\title{
Nephrotic Syndrome
}

National Cancer Institute

\section{Source}

National Cancer Institute. Nephrotic Syndrome. NCI Thesaurus. Code C34845.

A collection of symptoms that include severe edema, proteinuria, and hypoalbuminemia;

it is indicative of renal dysfunction. 